\title{
Editorial
}

\section{Advanced Materials and Technologies for Structural Performance Improvement}

\author{
Stefano Sorace, ${ }^{1}$ Bert Blocken, ${ }^{2,3}$ Claudio Borri, ${ }^{4}$ Luca Caracoglia, ${ }^{5}$ \\ Francisco Javier Molina, ${ }^{6}$ and Gerhardt Müller ${ }^{7}$ \\ ${ }^{1}$ Polytechnic Department of Engineering and Architecture, University of Udine, Via delle Scienze 206, 33100 Udine, Italy \\ ${ }^{2}$ Department of the Built Environment, Eindhoven University of Technology, P.O. Box 513, 5600 MB Eindhoven, Netherlands \\ ${ }^{3}$ Department of Civil Engineering, KU Leuven, Kasteelpark Arenberg 40, 3001 Leuven, Belgium \\ ${ }^{4}$ Department of Civil and Environmental Engineering, University of Florence, Via S. Marta 3, 50139 Florence, Italy \\ ${ }^{5}$ Department of Civil and Environmental Engineering, Northeastern University, 441 Snell Engineering Center, \\ 360 Huntington Avenue, Boston, MA 02115, USA \\ ${ }^{6}$ ELSA Laboratory, Institute for the Protection and Security of the Citizen, Joint Research Center of the European Commission, \\ Via E. Fermi 2749, 21027 Ispra, Italy \\ ${ }^{7}$ Department of Civil, Geo and Environmental Engineering, Technical University of München, Arcisstrasse 21, \\ 80333 München, Germany
}

Correspondence should be addressed to Stefano Sorace; stefano.sorace@uniud.it

Received 17 April 2016; Accepted 17 April 2016

Copyright (C) 2016 Stefano Sorace et al. This is an open access article distributed under the Creative Commons Attribution License, which permits unrestricted use, distribution, and reproduction in any medium, provided the original work is properly cited.

The development of advanced materials and technologies for application to new and existing structures, infrastructures, and equipment, aiming at improving their response to service and extreme loads, represents an emerging issue both from an academic and a professional viewpoint. Relevant performance assessment procedures, based on effective experimental verification methods [1] and refined numerical simulation models [2], are quickly evolving as well.

New or improved building materials and innovative protection technologies offer updated solutions to the constantly increasing capacity requirements in structural, geotechnical, plant, and mechanical engineering design and rehabilitation. At the same time, they open novel and challenging research and application perspectives.

The objective of this special issue was to provide readers with a representative outlook of the latest achievements in this field, including emerging aspects in modelling, testing, manufacturing, and practical implementation studies. The response of the scientific community was encouraging, with over 100 manuscripts submitted. The published papers offer a selected and articulated overview of the examined topics.

Several papers are dedicated to concrete, reinforced concrete (R/C) members, and cement composites. J. Zhang and J. Li develop a semi-implicit constitutive integration algorithm for elastoplastic damage models, specially implemented for concrete. Literature mechanical tests of reinforced concrete components are numerically simulated, showing the effectiveness of the numerical method in history-dependent energy estimation within this class of nonlinear problems. $\mathrm{H}$. Ding et al. examine the failure criteria and the constitutive relation of early-age concrete, with the aim of providing a theoretical basis for a novel strategy in early dismantling formwork construction of floors, aimed at accelerating formwork recycling as well as at reducing construction costs. Y. Shen et al. propose a paper spool-inspired anchoring method and a pretension procedure for carbon fiber reinforced polymer (CFRP) sheets, for the manufacturing of prestressed concrete flat slabs. Laboratory flexural tests confirm the feasibility of this novel reinforcement technique, showing that uniformity of the fiber stress during tensioning is a key aspect for the successful application of prestress. Improved flexural calculation models for $\mathrm{R} / \mathrm{C}$ members built with ultrahigh performance concrete (i.e., with cubic compressive strength exceeding $200 \mathrm{MPa}$ ) are presented by B.-I. Bae et al., who revise the normal stress distribution schemes adopted for standard concrete classes. The models are verified and validated by means 
of laboratory flexural tests on representative specimens in this case too. High strength concrete materials, with cubic compressive strength ranging from about $80 \mathrm{MPa}$ to $110 \mathrm{MPa}$, are examined in the paper of L. Zeng et al., as constituting materials of R/C beam-to-column joints encasing I-shaped hot-rolled steel profiles. An extensive campaign of cyclic tests on half-scale weak-type joints allows evaluating the beneficial influence of the adopted concrete classes in terms of strength and at the same time a minimal decrease in ductility, as compared to the cyclic performance of similar joints cast with standard concretes. Composite concretesteel tubular columns are the topic of the article by $\mathrm{S}$. Jayaganesh et al. An experimental programme is carried out to evaluate the effects of the partial compression of square and circular cross sections, highlighting a reduction in strength in comparison to full compression, more accentuated in the case of the square shape. The correlation of the test results with the predictions of the analytical models proposed in the literature to account for these effects seems rather satisfactory. Another experimental activity is reported in the paper by J. Deng et al., dedicated to a campaign of pseudostatic cyclic tests of one-fifth scale prototype $\mathrm{R} / \mathrm{C}$ bridge piers, damaged and repaired by means of a steel-tube jacketing traditional technique, or by wrapping interventions based on the use of CFRP or basalt fiber reinforced polymer (BFRP) sheets. The results attest satisfactory performance in all cases, with a significant increase in lateral stiffness and strength provided by jacketing as compared to the original response capacity, and its complete restoration in the case of the two FRP-based retrofit measures. The advantages of the latter method consist in lower physical intrusion and quicker installation times. The postprocessing study of an experimental programme carried out by the pseudodynamic method on a set of $13 \mathrm{R} / \mathrm{C}$ shear walls is presented in the article of F. J. Molina et al., where the results of the seismic tests are elaborated by an innovative strategy that allows deriving a capacity-line model. With this approach it is possible to understand better the different safety margins that are observed in the experiments depending on the assigned eigenfrequency in relation to the earthquake response spectra. The effects of the amount of reinforcement and normal load are also assessed. Enhanced cement composite materials are investigated in the articles by $\mathrm{H}$. Choi et al. and J. Lai et al. The former deals with the experimental characterization of an innovative Portland cement mortar, prepared by incorporating multiwalled carbon nanotubes in the mixture. The experimentally measured compressive strength is greater than the one offered by standard Portland mortars, as a consequence of the bridging action of the nanotubes, which prevents the progression of microcracks and helps reducing the space between the voids in the hydration material. In J. Lay et al.s paper, cement fly ash gravel is examined as constituting material of pile composite foundations, for their updated use in saturated tailing dams. A finite element analysis of the settlements of this type of foundation, calibrated on the experimental investigation carried out by other authors, assesses a stable and rather homogeneous response under the design embankment loads.

Three articles are dedicated to advanced seismic protection technologies. In the paper by M. Ismail et al., a numerical study of a base isolation system incorporating Roll-in-Cage devices recently developed by the authors is carried out by simulating its incorporation in a three-span prestressed $\mathrm{R} / \mathrm{C}$ box girder-type bridge. The results show a remarkable global performance of the system, also in comparison with other seismic isolation solutions, highlighting its benefits in the potential application to bridge structures. The article of F. Mazza and A. Vulcano reports a shaking table testing programme developed on a one-third scale base-isolated steel frame structure equipped with an in-parallel combination of steel-PTFE sliders and elastomeric bearings (HDRBs). The experimental response confirms the high level of protection offered by the isolation technology and establishes a database for a refined numerical modelling of the constituting devices. J. W. Hu and M.-H. Noh present a study on selfcentering friction dissipative steel braces including special components fabricated with shape-memory alloy wires and friction-type dampers. A numerical investigation carried out on single-degree-of-freedom models allows formulating a design methodology aimed at mutually optimising the recentering and energy dissipation capacities of the passive protection system, with a view to its practical implementation.

Two more papers deal with the application of innovative FRP composite materials in various fields of engineering. N. B. Baba et al. examine the mechanical and physical behaviour of a hybrid glass FRP (GFRP) fabricated with three different types of glass fibers-3D, woven, and choppedcombined with a mixture of polyester resin and hardener. Fiber arrangement and volume are varied to evaluate the combination capable of providing the best performance in terms of tensile strength and water absorption, which is identified by means of the characterisation test developed in the study. In the article by M. E. Taşdelen et al. the torsional behaviour of novel braided sleeve composite shafts consisting of carbon and glass fibers is investigated. Vacuum assisted resin transfer molding and vacuum bagging are adopted as manufacturing methods. The results of the experimental programme and the relevant finite element simulations underline that, for the same configuration of layup sequences, the highest torque and tensile strengths are obtained for test specimens manufactured with the former method. Furthermore, hybrid carbon-glass solutions strike the best balance between strength performance and costs.

Special issues are dealt with in the remaining three articles. By considering the growing applications of piezoelectric structures in innovative technical areas, L. M. Zhou et al. implement a mixed cell-based smoothed/extended finite element method for a careful electromechanical crack analysis of the constituting material. The case study examples presented in the paper show that this mixed method is more accurate and quickly converging than a standard finite element calculation, also bypassing the mapping process inherent to the latter, which significantly increases the complexity of the calculation. J. Chen et al. report an on-site experimental study of the cooling effect of crushed rock-based embankments on slope wetlands situated along a railway line. A monitoring system installed in an embankment of notable dimensions allows reconstructing the temperature fields and the freezing 
history with time. The surveyed data show that only the embankment shady side can be effectively cooled down in a warm permafrost region, and the crushed rock-based embankment can cool the entire embankment in a cold permafrost region. Finally, in the paper by Y. Ou et al. an innovative self-propagating high-temperature synthesis (SHS) process to prepare amorphous boron powder, which is an attractive material in several enhanced engineering applications, is discussed. The new technique proposed in this study consists in combining the SHS process and the traditional magnesium thermal reduction method. The effectiveness of the resulting technique suggests its possible industrial application and adoption as a common approach to prepare various inorganic materials.

\author{
Stefano Sorace \\ Bert Blocken \\ Claudio Borri \\ Luca Caracoglia \\ Francisco Javier Molina \\ Gerhardt Müller
}

\title{
References
}

[1] S. Sorace and G. Terenzi, "The damped cable system for seismic protection of frame structures. Part I: general concepts, testing and modeling," Earthquake Engineering \& Structural Dynamics, vol. 41, no. 5, pp. 915-928, 2012.

[2] S. Sorace and G. Terenzi, "Structural assessment of a modern heritage building," Engineering Structures, vol. 49, pp. 743-755, 2013. 

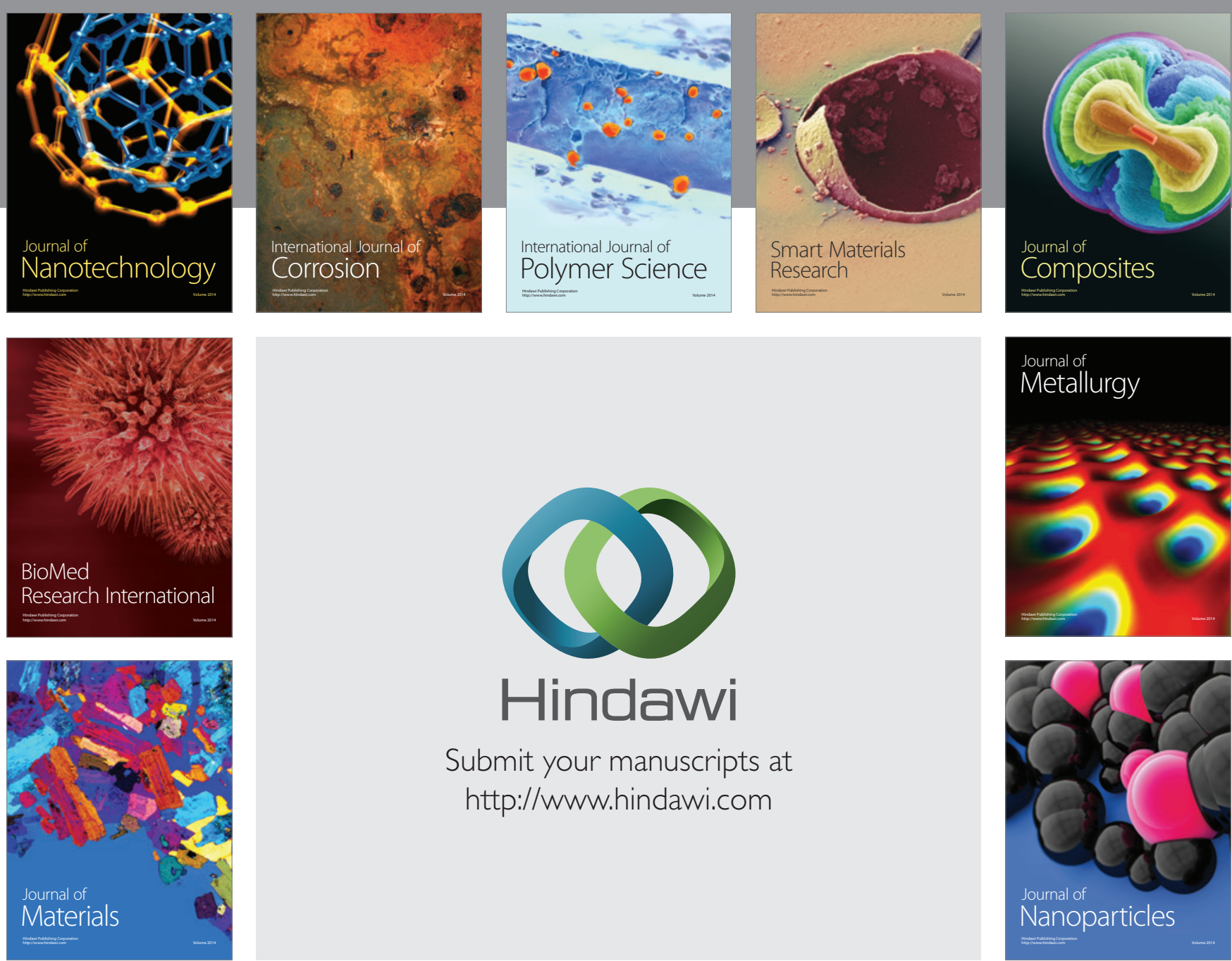

\section{Hindawi}

Submit your manuscripts at

http://www.hindawi.com

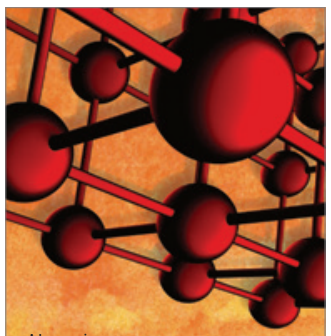

Materials Science and Engineering
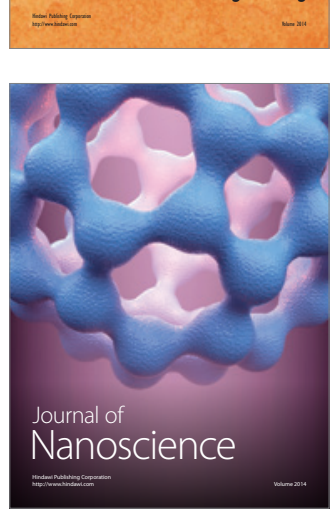
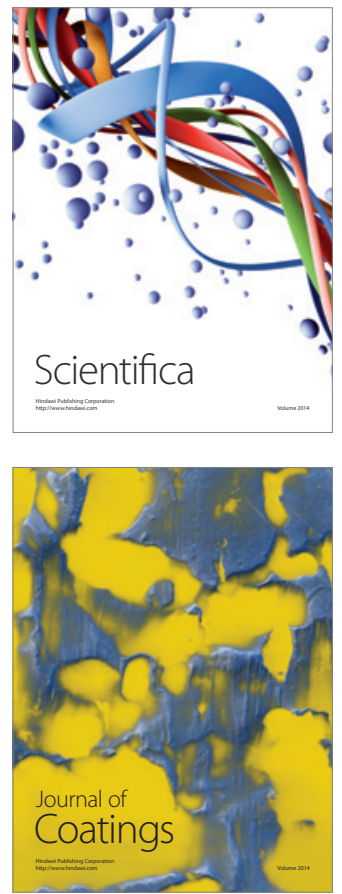
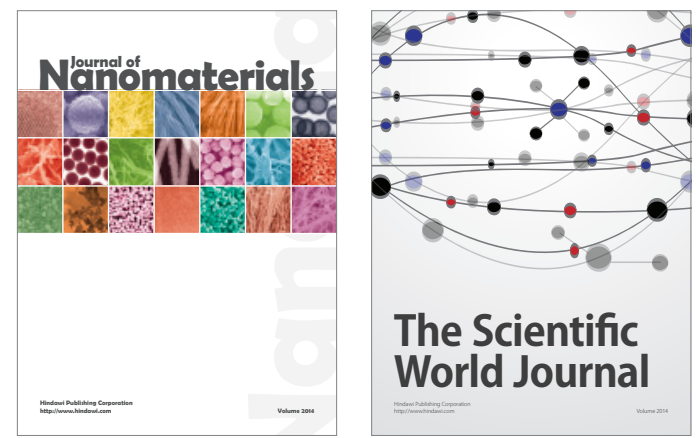

The Scientific World Journal
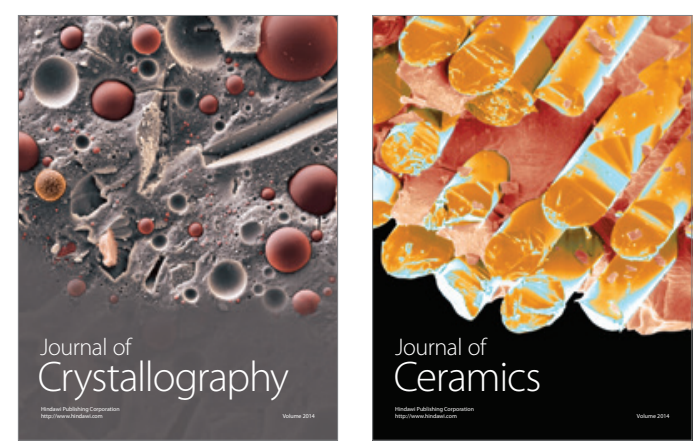
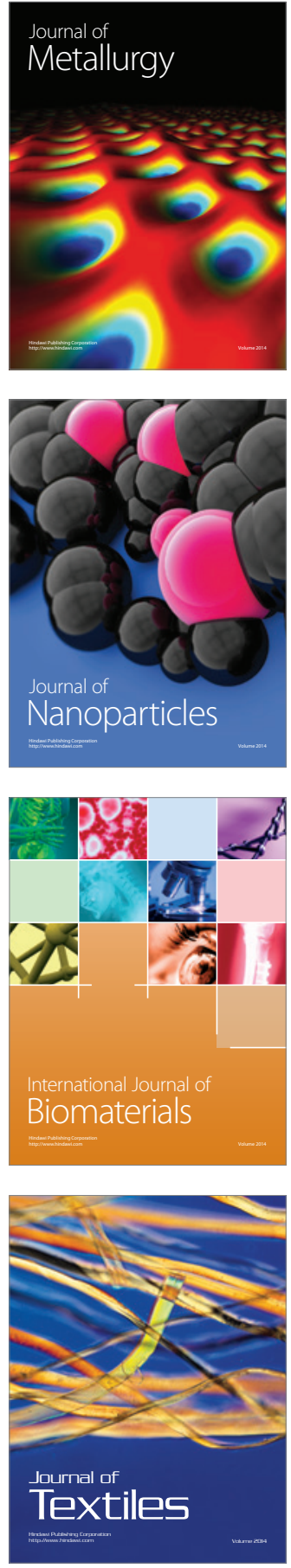